Uwe Scheffler

\title{
ON THE LOGIC OF EVENT CAUSATION
}

Part I

Fundamental Reflections

Received August 2, 1993 


\begin{abstract}
The paper outlines an analysis of token causation. It contains reasons for the choice of event tokens and a list of properties of causal relations. After defining the notion event it is shown, in which form classical philosophical problems arise and how they can be solved: Causality is interpreted as an second order property of first order relations. Temporal order can be induced from causal order. Token causation does not involve neither any kind of necessity, nor probability. Causal laws are generalizations on sentences about token causation by quantifying over event types. Two explications of Causal law are suggested, differing in the ability of allowing for exceptions. ${ }^{1}$
\end{abstract}

\title{
CONTENTS
}

1. Once again causality — why?

2. Structure

3. Events

4. The relation - some properties

5. The relation - some ideas

6. Realism?

7. Time

8. Necessity and probability

9. Laws and explanations

References

\footnotetext{
${ }^{1}$ I am indebted to the Fritz Thyssen Stiftung for a grant which made possible my work at the University of Konstanz during 1991 - 1993, and to the Deutsche Forschungsgemeinschaft for supporting a common larger project of MAX URCHS and me on Causal Theory. I am grateful to Weyma Lübbe, Ingolf Max, Hans Rott, and Max Urchs for conversations relating to the topic of this paper.
} 


\section{Once again causality - why?}

There is no undisputed theory of causality as well as there is no paradigmatic system of causal logic (as there are, for example, the LEWIS-systems for modal logic or $\mathrm{E}$ and $\mathrm{R}$ for relevant logic). The latter is, of course, mostly due to the former; causal logic, if possible at all, is possible only as a kind of twice applied logic: Since causal relations are not logical relations, the logic of causal relations should include at least some extra-logical, contingent from the point of view of pure logic elements. It depends on a causal theory. Since causal logic aims to support, measure, check or destroy causal theories, it should be applicable to a variety of examples, often stemming not from logic or philosophy, but from everyday life or empirical sciences. The absence of a generally acknowledged theory of causation appears to be an unavoidable disaster, because Causality is an umbrella term, covering several different ideas about dependencies. The disaster is unavoidable, because people really use causal terminology in order to describe dependencies of different types, hence different causal theories (and, in continuation, "causal logics") are legitimate and reasonable. However, it seems to be legitimate and reasonable too, to ask whether there is (or are) a (or some) basic approach to a causal terminology, basic in at least two senses: basic because it allows to introduce a lot of different causal terms; and basic because it describes some fundamental kind of causation, as plain and straightforward as possible at all. The answer to this question is, in my opinion, Yes, and the present paper is almost nothing more than a detailed argument about why I should believe so.

The first suggestion is to differentiate between the following questions, sometimes mixed up at the very beginning of the analysis:

1. What is the logical structure of a causal sentence? That means, what kind of entities are connected by Causality - things, events, propositions, states of affairs, or others, and how to represent the causal nexus itself - as a logical connective, a function, an unary, binary, or ternary relation, or as something else?

2. What are the truth-conditions for a causal sentence? This question is connected with the modal state: are there logical or nomological relations between cause and effect, is a causal sequence an observable, a contingent fact?

3. What are the circumstances, which allow to identify causal sequences as causal? This question mostly deals with epistemological problems 
and should be answered in a context of regularity, probability, and time.

It is worth mentioning, that there is also an interrelated psychological aspect, properly being called the disaster theory of causation. This theory is grounded on the observation, that people mostly ask for causal explanations (ask "why?") in individual situations in which something occurs differently from what is expected to occur:

In ordinary life the particular causal question is most often inspired by the wish for an explanation of a particular contingency the occurrence of which is puzzling because it is a departure from the normal, ordinary, and reasonably expected course of events: some accident, catastrophe, disaster or other deviation from the usual course of events. ([6], p. 31)

HART and HONORÉ are perfectly right in pointing out a fact, which corresponds to theories of singular causation: causal relations are used to explain causally the occurrence of singular events. Of course, if experience is something like a continuous movie from which people somehow cut slices named events, especially unexpected parts of the movie call for explanation. Nevertheless, the attempt to include this idea into the very notion of causation (cf. [13] for a survey) meets a lot of difficulties: It is rather a psychological than an ontological, semantic or epistemic question whether an event is unexpected or not. The answer to this question depends not only on what the person who asks it knows about laws and regularities, but also on what she/he is currently able to see and hear, and on which temperament she/he possesses. There should be a definition of what is meant by expected or the usual course, without recourse to causality. Besides all theoretical arguments a short examination of real cases shows, that people often are interested in "ordinary" causal sequences, for example, in order to be able to change the next occurrence of the sequence.

The "disaster theory of causation" confuses the question when a causal explanation is sensible, meaningful, and necessary, with one or more of the questions mentioned above. Nevertheless, it contains a fruitful basic idea: people have some freedom in choosing which events are to be analyzed causally. 


\section{Structure}

Judgements about causal dependencies could be expressed by several means. Let us consider the very general and probably undoubted expression "An $A$ is the cause of a $B$ ", then the problem to be solved consists in two questions: What are the " $A$ " and the " $B$ " standing for? What is the "is the cause of" standing for? Ordinary language and logical analysis provide a lot of possibilities to answer the questions in different manner; the one preferred here is the following: Causality is a binary relation between singular events (event tokens).

Why singular events (event tokens), and not event types? Event tokens are abstract particulars; abstract because at the same spatiotemporal region more than one event token can occur, particulars because they are localized in space and time. Event types are universals, having appropriate tokens as instances.

Given a suitable understanding of token causation and a theory of events it seems to be natural to handle type causation as a quantificational extension of token causation - just as in other cases of "induction": All observed particulars of a certain kind have a certain property, hence we (hypothetically, for the beginning) conclude, that all particulars have the property, and often express this by a sentence about the kind.

A lot of problems with causation arise only on the type causation level, because tokens do not recur. Starting with token causation opens the chance to find out, which of the problems descend from causality, which from quantification theory and which are due to the common problems of induction.

Using event tokens allows to understand causality as to be concerned with empirical entities, hence we will have a settled understanding of causality when the talk goes on to causal laws and probability. Type causation without an earlier founded token causation requires an answer to the question, how we could know about a non-logical relation before knowing empirical facts.

It seems to be not to easy to distinguish consequently between token and type causation. Typically mistakes are made in discussing formal properties of the causal relation. Sometimes even after explicitly claiming the usage of tokens arguments like the following could be found:

Note that if $A_{i} \cap B_{j}$ is a cause of $C_{l}$, it does not automatically follow that factors $A_{i}$ and $B_{j}$ are also causes of $C_{l}$. It may be true that taking medicines $a$ and $b$ together made me feel bad, but it may be false that if I had not ta- 
ken medicine $a$ alone I would not have felt bad, and also false that if I had not taken medicine $b$ alone I would not have felt bad. ([10], p. 150)

Token causation does not allow such kind of counterfactual reasoning the event $A_{i} \cap B_{j}$ happened, and therefore the events $A_{i}$ and $B_{j}$ happened too. If the first event is the cause of $C_{l}$, none of the latter events could be considered standing in a single causal connection to the effect, because this would constitute other, completely different causal sequences. In the given circumstances both $A_{i}$ and $B_{j}$ are causes of $C_{l}$, because there are no other circumstances (by definition) and they occur together.

Why singular events, and no other kind of entity? Natural language allows to use expressions for other entities in causal sentences, such as for physical things or even moral or psychical states: "That bullet is the cause of his death", "His stinginess is the cause of his small tips". Taken that literally, we should assume something like Aristotle's material cause, but such an understanding of causality seems to be quite unusual today. The obvious solution consists in interpreting causal sentences of this form as elliptical speech, as abbreviations for sentences about events.

Things or moral states are not the natural rivals for events in causal dependencies. Instead, promising candidates are either facts or states of affair, or certain kinds of events like actions or changes. Actions and changes can be causes and effects, but are not the only kind (or kinds) of causes and effects. As a matter of fact, I believe, there is causality out of the area of actions, and sometimes just a non-change is a cause of somewhat else. The difference between events and facts is often not very far and depends on appropriate definitions. In the framework of the proposed approach the main difference consists in events being entities somehow connected with the empirical world (what will be explained later), while there are logical, mathematical, moral, ..., facts. States of affair generally include more, than is wanted for causality: events are specific (small or big) pieces of reality. An outline of a theory of events is given in the next section.

Why a binary relation? Most work in causal logic was done by introducing or defining a causal connective, either an (usually) unary operator of causal necessity, or, more often, an (usually) binary causal implication. Because the connective is defined over sentences and not event terms or even events, the analysis deals with event constituting sentences or introduces an occurrence operator, producing sentences from event terms (names of events). I prefer to use a relation instead of a connective only for the sake of style: while logical connectives and their properties are a matter of language, 
causal relations and their properties are on the whole a matter of fact and philosophy. Causal implications taste much more similar to material, modal, relevant, and other implications, than causal relations do.

Why a binary relation? The straightforward approach to causality, of course, leads to binary relations. It can be replied, that any judgment about a causal dependency includes a "hidden variable": the relevant conditions, under which the cause could have caused the effect:

\begin{abstract}
Whereas causality is normally conceived as a relation between a cause and its effect, the idea is to represent causality as a predicate which takes three arguments. The point is that there is always a ceteris paribus clause involved in a statement regarding causality. This clause determines which possible histories should be taken into consideration in order to evaluate the statement in question. In other words, the third argument of the causality predicate defines the set of entities relevant for the evaluation of the statement. ([7], p. 11)
\end{abstract}

There is no need for ceteris paribus conditions in token causality. The causes I am interested in are partial causes as opposed to complete causes, hence in any case it is possible to recognize the existence of not only "necessary" conditions, but also of further causes without rejecting the initial causal statement. Because causes and effects are not necessary unstructured (in fact, in most cases they are compound), it is possible to grasp the events as complicated, as necessary.

\title{
3. Events
}

To obtain a theory of events suitable for causal theory I assume linguistic conventionalism with respect to events, the idea, that events are language-dependent entities. VAN BENTHEM expresses this view by:

\footnotetext{
It is obvious that events are linguistic constructions. Experience is a continuous movie, which humans cut into manageable slices using their language. ([3], p. 113)
}

If this is right, two conclusions should be drawn: What events human beings get depends on the language actually used and on human aims and intentions; the ontological question of what events are changes to the terminological question of how to manage event terms. At the other hand it is possible to take part in events, to see events. Hence, even if events are not 
ontologically independent, it is necessary to show on which entities they are supervenient.

Most of our sentences are understandable only in a context of conversation. Often this context contains especially the reference to a spatiotemporal region or to concrete objects, to which general terms refer. It is possible to map all context dependent sentences (with respect to spatiotemporal regions and general terms) into independent sentences using the following two steps:

Regard all sentences as completed by constants or bound variables for spatiotemporal regions as far as they are (in this respect) understandable by themselves. These sentences are universal in contrary to local sentences (cf. for more detailed information [17], pp. 496f.).

Regard all sentences containing free general terms as completed by special quantifiers over general terms; the resulting sentences are singular in contrary to general sentences.

Spatiotemporal regions and general subject terms will play an decisive role in the construction of event types. It is therefore necessary to use a language, which includes at least the following parts:

variables for spatiotemporal regions $l, l^{1}, l^{2}, \ldots$

constants for spatiotemporal regions $t, t^{1}, t^{2}, \ldots$

quantifiers for spatiotemporal regions $\forall, \exists$

singular subject terms $v, v^{1}, v^{2}, \ldots$

general subject terms $w, w^{1}, w^{2}, \ldots$

quantifiers for general subject terms $\Sigma, \Pi$

predicate terms $P, P^{1}, P^{2}, Q, Q^{1}, Q^{2}, \ldots$

truth-functional operators $\sim, \vee, \wedge$

a conditional operator $\longrightarrow$

the termforming operator $t^{*}$, forming names of sentences or terms from sentences or terms

the constant predicate of meaning inclusion between terms $\rightarrow$

the constant predicates of positive and negative predication $\leftarrow$ and $\nleftarrow$

Intuitively meaning inclusion means that all, what is named by the first term, is also named by the second term; it holds either between subject terms or between predicate terms, but not between a subject and a predicate term. Positive predication means that an object has a property (or objects are standing in a relation), negative predication means that the property (or relation) is denied of the object (the objects). Obviously there is a definable 
third kind of predication: It is not true that the object has the property and it is not true that the property is denied; this is uncertainty predication.

The next step is the introduction of a new termforming operator $s$, forming event terms from universal and singular sentences:

Definition 1. The term $s A$ is an event term if and only if $A$ is a universal and singular sentence, and

1. all terms in $A$ are empirical terms; and

2. A is neither logically or analytically true nor logically or analytically false.

It is easy to see that all event terms obtained according to this definition describe event tokens: concrete, singular, spatiotemporally localized events. This is due to the fact, that all sentences refer to a concrete spatiotemporal region (as big as it may be), and to concrete individuals or explicitly to some or all individuals of a type. Given these singular event terms it is possible to construct terms describing event types (general event terms), that means: schemes, types, kinds of events. The following constructions allow to understand the relation between singular event terms and appropriate general event terms as a relation of meaning inclusion. Furthermore, after introducing general event terms it seems natural to understand singular events as particular instances of universal event schemes. The main idea of the following rules is to weaken the conditions on "universal and singular sentence":

Definition 2. The event term $s B$ is an event scheme for $s A$ with respect to spatiotemporal regions if and only if $s B$ can be obtained from $s A$ by erasing 0 or more occurrences of spatiotemporal constants and variables (with their quantifiers) in $s A$.

Definition 3. The event term $s B$ is an event scheme for $s A$ with respect to subjects if and only if $s B$ can be obtained from $s A$ by erasing 0 or more occurrences of term quantifiers in $s A$ or by substituting subject terms $\left(u^{a^{1}}, u^{a^{2}}, \ldots\right)$ by subject terms $\left(u^{b^{1}}, u^{b^{2}}, \ldots\right)$ in $s$ A such, that $t^{*} u^{a^{1}} \rightarrow t^{*} u^{b^{1}}$, $t^{*} u^{a^{2}} \rightarrow t^{*} u^{b^{2}}, \ldots$

Definition 4. The event term $s B$ is scheme for $s A$ if and only if it is possible to construct a finite number of event terms $s B^{1}, s B^{2}, \ldots, s B^{n}$, and it holds: $s B^{1}$ is scheme with respect to spatiotemporal regions or with respect to subjects for $s A, s B$ is scheme with respect to spatiotemporal regions or 
with respect to subjects for $s B^{n}$, and all neighboring constructed event terms are in one of these relations.

Observe, that there are schemes of schemes, and that the relation of being a scheme for is reflexive, nonsymmetric, and transitive.

Two axioms should be added:

Terms describing identical events (whatever this is) have the same instances and the same schemes.

If $s B$ is scheme for $s A$, then $t^{*} s A \rightarrow t^{*} s B$.

The first axiom makes the relation of being a scheme for independent of the concrete linguistic form of the given event terms. The second axiom guarantees the possibility of term quantification about general event terms by extending the relation of meaning inclusion to event terms.

The whole construction amounts to introduce events to ontology (a more detailed version of the theory of events used here is outlined in [11] and [12]):

Definition 5. An event is, what is described by an event term.

In more detail:

An event token is, what is described by a singular event term.

An event type is, what is described by a general event term.

Definition 6. An event token sA exists if and only if the sentence $A$ is true.

An event type $s A$ exists if and only if there is an event token $s B$ such, that $t^{*} s B \rightarrow t^{*} s A$ and $s B$ exists.

The second part of the definition is quite natural and clear, kinds of events are existent if there is at least one particular existing instance. The first part of the definition says that the existence of events depends on exactly the same, on which the truth of the constituting sentence depends.

Obviously existing events could play the role of truth-makers: A sentence is true, if and only if it constitutes an existing event follows from the definitions. The existence of events is not independent, neither of language, nor of the given object ontology. Not only the expressive power of languages determines, which events exist, but also some logical features. Sentences, constituting existing events in a language, may for example turn out to be analytic in another language and therefore do not constitute events at all. The dependence on object ontology is evident, because of the dependence of truth on the existence of certain things. 


\section{The relation - some properties}

This part of the paper aims to clarify, which formal properties a causal relation should have. The more philosophical properties are discussed in the following parts of the paper. Since we are still not yet in causal logic (remember the subtitle: Fundamental reflections), the arguments are based on intuitive considerations. The selection of principles cannot be complete, but representative in the sense of containing most of the everywhere discussed properties.

In order to simplify the notation, $\alpha, \beta, \ldots, \alpha_{1}, \ldots$, are used as singular terms, standing for event tokens. An expression like $\alpha \wedge \beta$ then stands for $s(A \wedge B)$, or, given a suitable termforming conjunction, for $s A \dot{\wedge} s B$. Clearly, not all subformulas of an event constituting formula constitute events on their part, as well as not all truth-functional combinations of event constituting formulas are event constituting. Consequently, combining or partitioning events requires a "safety test" by definitions 1 and 5 , whether the resulting entities are events at all. In the following is assumed, that they are. Further, let $\mathcal{U}$ be the binary predicate "is a cause of".

The first question to be answered is: which influence have logical operators on causes and effects?

\section{Conjunction}

$$
\text { If } \mathcal{U}(\alpha \wedge \beta, \gamma) \text {, then } \mathcal{U}(\alpha, \gamma)
$$

A counterfactual objection to this principle was already mentioned: it could easy be, that $\alpha$ alone, without $\beta$ would not have caused $\gamma$. Nevertheless, by assumption $\alpha$ occurred together with $\beta$, hence in that special (token) situation $\alpha$ was a cause of $\gamma$. There are strong epistemic reasons for denying the principle: people often are not able to select a "small" cause at the very beginning of the analysis. They start with an event and then, step by step, exclude the causally irrelevant parts, arriving at smaller events. In such cases it would be unreasonable to have that principle. Further, events can be described by different ways, so some descriptions may include causally irrelevant parts (for pragmatic reasons, for example). It has to be mentioned, that in idealized situations where such "failures" do not occur, and in the absence of monotonicity the principle can be accepted.

$$
\text { If } \mathcal{U}(\alpha, \beta \wedge \gamma) \text {, then } \mathcal{U}(\alpha, \gamma)
$$


In this case, like in the former, people should be allowed for starting their analysis with bigger effects, and then reducing them by dropping causally irrelevant parts. This could not be done in the presence of the principle in question.

$$
\text { If } \mathcal{U}(\alpha, \gamma) \wedge \mathcal{U}(\beta, \gamma) \text {, then } \mathcal{U}(\alpha \wedge \beta, \gamma)
$$

If an effect has several different causes, this principle allows for summarizing them into a structured bigger event. Usual objections, based on the possibility of interfering, counteracting causes are neglectable - they do it only in the type causation case.

$$
\text { If } \mathcal{U}(\alpha, \beta) \wedge \mathcal{U}(\alpha, \gamma) \text {, then } \mathcal{U}(\alpha, \beta \wedge \gamma)
$$

Different effects of a cause also can be summarized in a single effect event. Of course, sometimes such effect events turn out to be unusual large and complicated.

\section{Disjunction}

$$
\text { If } \mathcal{U}(\alpha \vee \beta, \gamma) \text {, then } \mathcal{U}(\alpha, \gamma) \vee \mathcal{U}(\beta, \gamma)
$$

The existence of disjunctive events is controversial. Without further discussion and argument I indicate two cases, in which people make use of disjunctive events: in situations of incomplete knowledge, where it is unknown which alternative really came true; and in case of events, which are identical with a disjunctive event (drawing a card from a stock of black and red cards, for instance). Therefore it seems to be plausible to accept the principle, just like the following one:

$$
\text { If } \mathcal{U}(\alpha, \beta \vee \gamma) \text {, then } \mathcal{U}(\alpha, \beta) \vee \mathcal{U}(\alpha, \gamma)
$$

The next two principles are of special interest because with their help it is possible to connect completely different events in a causal structure, and because it is necessary to check the "disjunctive entities" whether they are events:

$$
\begin{aligned}
& \text { If } \mathcal{U}(\alpha, \beta) \text {, then } \mathcal{U}(\alpha \vee \gamma, \beta) \\
& \text { If } \mathcal{U}(\alpha, \beta) \text {, then } \mathcal{U}(\alpha, \beta \vee \gamma)
\end{aligned}
$$

It seems to do no harm to include both that principles in the list of accepted principles under the condition, that the disjunctive events are events. Of course, they are rather useless. 


\section{Negation}

$$
\text { If } \mathcal{U}(\sim \alpha, \beta), \text { then } \sim \mathcal{U}(\alpha, \beta)
$$

By $\sim \alpha$ are indicated "negative events", corresponding to appropriate "positive events". So if $\alpha$ is $s A, \sim \alpha$ is $s \sim A$. Negative events are at least as disputable as disjunctive events (arguments for negative events are listed in [11], pp. $12-15)$. Once taken for granted, that there are such strange entities like negative events, the principle mentioned above must be accepted: if $\sim \alpha$ exists, $\alpha$ does not exist, hence it cannot be a cause. The same holds for effects:

$$
\text { If } \mathcal{U}(\alpha, \sim \beta), \text { then } \sim \mathcal{U}(\alpha, \beta) .
$$

Obviously, both principles hold only in the indicated direction.

\section{Conjunction and disjunction}

$$
\begin{aligned}
& \text { If } \mathcal{U}(\alpha, \beta \wedge \gamma) \text {, then } \mathcal{U}(\alpha, \beta \vee \mathcal{U}(\alpha, \gamma) \\
& \text { If } \mathcal{U}(\alpha \wedge \beta, \gamma) \text {, then } \mathcal{U}(\alpha, \gamma) \vee \mathcal{U}(\beta, \gamma)
\end{aligned}
$$

Both principles are acceptable, what becomes clear after observing, that they are weaker variants of the first two discussed principles.

The next few principles are usually discussed in connection with causality:

\section{Irreflexivity}

$\sim \mathcal{U}(\alpha, \alpha)$

This principle excludes the possibility of a causa sui. It is nowadays undisputated.

\section{Antisymmetry}

$$
\text { If } \mathcal{U}(\alpha, \beta), \text { then } \sim \mathcal{U}(\beta, \alpha)
$$

Antisymmetry prevents the occurrence of direct loops. As it becomes clear in section 7 , it is reasonable to prevent also indirect loops.

\section{Non-transitivity}

It is not the case, that if $\mathcal{U}(\alpha, \beta) \wedge \mathcal{U}(\beta, \gamma)$, then $\mathcal{U}(\alpha, \gamma)$ 
Often transitivity is accepted in causal theories. Nevertheless, there are interesting and strong counterexamples, and non-transitivity seems to be the natural way of how causal chains break. Needless to remember, that causal chains may exist under non-transitivity.

\section{Monotonicity}

$$
\text { If } \mathcal{U}(\alpha, \beta) \text {, then } \mathcal{U}(\alpha \wedge \gamma, \beta)
$$

Monotonicity can be tolerated, if $\alpha \wedge \gamma$ is an event and exists. This is due to the rejection of the first principle ("Conjunction"), so it is not allowed to conclude $\mathcal{U}(\gamma, \beta)$ from $\mathcal{U}(\alpha \wedge \gamma, \beta)$. It is a matter of taste, whether one likes to accept monotonicity or not, and rather of formal, than of practical interest.

It is not the case, that $\mathcal{U}(\alpha, \beta) \vee \mathcal{U}(\alpha, \sim \beta)$

In analogue to conditionals this principle may be called the denial of the causal excluded middle, it obviously holds due to the possible nonexistence of $\alpha$.

It is not the case, that $\mathcal{U}(\alpha, \beta) \vee \mathcal{U}(\beta, \alpha)$

The principle tells, that causality is an unconnected relation on events. It is part of the intuition about causality, that there are events, which are not causally dependent - so the principle should be accepted even if related to all existing events.

An unsolved question is that about iterations of causal relations. Does it make sense to discuss principles like:

$$
\text { If } \mathcal{U}(\alpha, s(\mathcal{U}(\beta, \gamma))) \text {, then } \mathcal{U}(\beta, s(\mathcal{U}(\alpha, \gamma))){ }^{2}
$$

I would like to answer the general question with it depends and not to discuss principles with iterated causal relations here and now. It depends on the preferred understanding of the ontological status of events: the depicted approach to events suggests events to be parts of the empirical world. Hence, event terms can be considered as empirical terms, and $s(\mathcal{U}(\alpha, \gamma))$ is an event term by Definition 1. In this case, we have to allow for iterated causal relations. Conversely, if events are pure epistemic constructions or something else, what is not part of the empirical ontology, expressions like $s(\mathcal{U}(\alpha, \gamma))$

\footnotetext{
${ }^{2}$ (cf. [16], p. 5)
} 
cannot be subjects in causal sentences. The consequences of such an understanding of events can be exemplified with another observation: Suppose we want to have an operator $O$ - "occurs" (like in [14]), taking event terms to sentences. Then with both ontological interpretations of events we have

$$
A \longleftrightarrow O(s A),
$$

but only with the first we get

$$
s A \rightleftharpoons s O(s A) .
$$

\title{
5. The relation - some ideas
}

According to the outlined analysis causes and effects are particulars in their full concrete realization in space and time. Nevertheless, there are certain aspects of the cause, which bring about the effect, others are more or less causally irrelevant. Because causes usually have more than one effect, and effects more than one cause, even with respect to only one event different mechanisms of causation are observable. Causation is considered as a relation, but where is the causal emphasis? What makes the cause be a cause - is it a property of the event, or a property of the objects, involved in the event, a nomic relation between universals, participating in the process of causation, or something else? The answer to that question should be compatible with the view, that token causation is ontologically and methodologically prior to natural laws and type causation. As starting point serves a quotation:

\begin{abstract}
Explanation of an effect normally demands that an event be singled out as the cause, but further, it demands that the salient property of the cause be identified. As it is in ordinary language, so is it in scientific explanations where causes are identified. Thus it is the mass of the object in the scale pan that caused it to fall; it is the charge of the particle that caused it to be deflected by the magnetic field. Again, other properties may be quite irrelevant. The color of the object in the scale pan is irrelevant, the momentum of the deflected particle is irrelevant. Only certain properties of the causal events are relevant to the events as causes. (And, we may add, only certain properties of the effect are relevant as the effect of this cause.) ([8], p. 68)
\end{abstract}

First of all it attracts attention that the emphasized (by the authors) entities mass and charge are by no means properties of events. They are properties 
of objects, involved in events: in putting an object on the scale pan, respectively in conducting a particle through a magnetic field. On the other hand, these properties really play an important role: there are laws and functional dependencies allowing for calculating how much the scale pan falls given a certain mass, and how much the particle is deflected given a certain charge. These laws and dependencies are true independent of occurring events, and they still remain true, even if in a particular case an object suddenly behaves otherwise. No need for causality.

What properties of, or relations on events can we have? Refrained from human attitudes (expected/unexpected, wanted/unwanted, ...) we have temporal and local order, we can define a "part of" relation independent from temporal and local order, and ontological dependency. Ontological dependency exists also between other particulars: between a craftsman and her/his product, between an agent and her/his action, between a mathematician and a solution to a particular problem, between a physical object and a particular piece of material, from which it is made of, an event and the objects, involved in it, .... Ontological dependency between events can be arranged by several ways: by material forces (like masses in a gravitational field), by chemical processes, human actions or even mental influence, for instance.

Causality is here supposed to be the most important (but not the only one) kind of ontological dependency between event tokens. This explains differences and similarities between token and type causation: as it was already claimed, a type exists if a corresponding token exists, hence the only ontological dependency between event types could be that of both being dependent on certain tokens. It explains further, why it is reasonable to deal with incomplete, partial causes, and not with "The Cause": events may be ontological dependent from a lot of other events by different ways, so an entity like "The Cause" simply may not exist. It also explains, why people sometimes feel the strong influence of other entities, like masses in the quotation, because some events are ontological dependent on them, and others too, so there is a derived dependence, which is not necessary causal.

Since the relation is restricted to ontological dependencies, the following conditions are accepted in order to exclude other influences:

If $\alpha$ is part of $\beta$, then $\sim \mathcal{U}(\alpha, \beta)$ and $\sim \mathcal{U}(\beta, \alpha)$.

If $A \longrightarrow B$ is logically or analytically true and $A$ and $B$ constitute $\alpha$ and $\beta$ respectively, then $\sim \mathcal{U}(\alpha, \beta)$ and $\sim \mathcal{U}(\beta, \alpha)$. 


\section{Realism?}

The material of the previous section allows for a slightly different interpretation of causality. Assume, that event tokens are part of the empirical world, being supervenient on the existence of certain things and on relations between them, being also dependent on peoples wishes and intentions. Some pairs of such events stand in relations, which include a time ordering and are characterized by several forms of ontological dependence - usually expressed by causative verbs like "kill", "incite", "deflect", or "destruct" (cf. for causative verbs [1]). Really, "kill" then is a set of pairs of events, in which in the first event someone does something, and in the second event someone is dead; "incite" is a set of pairs of events, where in the first event someone is told to do something, and in the second event she/he does it; .... These relations are the ontological background of causality, and they are often observable.

Neither counterfactual analysis, nor regularity theory, nor probabilistic approach can explain, why token causality is in fact often observable. It is observable in just the same sense as, for instance, redness: sometimes people observe it correct, sometimes they are wrong, and sometimes they could not make any claim because of disadvantageous conditions:

\footnotetext{
A small piece of stuff is observed to be dropped into a glass of liquid, in a laboratory, say. The next thing perceived to happen is that the glass explodes violently. Under these circumstances we would have little doubt that we had witnessed a particular causal sequence. The dropping of the stuff into the glass caused the explosion. (An example of GASking, cited after [2], p. 93.)
}

The existence of those empirical relations between events on the bottom of the (theoretical) causal terminology explains the observability of token causation immediately.

Two possibilities of explicating causality arise from this approach: causality might be the union of all that other relations, or it is a property of those relations. In the first case causality is a first order relation between events, in the second it is a second order property: the universal "to be causal", being instantiated by first order relations. Clearly, who likes to have causality as an empirical relation, should either choose the former possibility, or be on quite strong realistic positions about second order properties. The view, that causality is defined on empirical entities, but itself depends on human activities (whether people look for causality, which parts of the future seem to be manageable, which relations people decide to understand as causal 
ones, ...), is compatible with both possibilities. It seems to be an pleasing position to choose the second order property view, to remain skeptic about second order properties, but to be on cautious realistic positions about the first order relations (let them be, for instance, not only sets of pairs of events, but something like processes).

In section 4 Transitivity was not included in the list of accepted principles. Causal chains sometimes break, what prevents causality from excessive expanding:

If, for example, a sensational, because rarely observed injury of a worker occurs due to the recklessness of the manager of a building site, which the treating surgeon describes in specialized literature, from legal position there is causal connection between the former two events, whereas from medical point of view the rare injury caused the publication. However, the recklessness of the manager is irrelevant for the physician, and from the publication of the physician rise no problems for the manager at his court hearing. ([15], p. 35)

Obviously the links, where the chain breaks, are that which link different basic causal relations, as it is mentioned in the example above.

\section{Time}

The causal relation is a directed relation: it is irreflexive and antisymmetric. The relationship between time order and causal order is a classical topic of causal theory and gives rise to a lot of questions, nevertheless I want to focus on the following:

1. Is it necessary, that cause and effect overlap in time? Do they?

2. Is it possible, to keep apart causal and temporal order? Does backward causation exist?

3. Does causal order induce a temporal order?

Causes and effects are particulars, instantiated at certain time intervals. The argument, which supports the claim, that these intervals should overlap, or at least pass into each other without gap, goes as follows:

1. If $\mathcal{U}\left(\alpha\left(t^{1}\right), \beta\left(t^{2}\right)\right)$ and $t^{1}$ ends earlier, than $t^{2}$ begins, then in the meantime there should be an event, which causes the occurrence of $\beta\left(t^{2}\right)$ just at 
the time it occurs. Hence, we have to consider the third event to be the cause of $\beta\left(t^{2}\right)$.

2. In the meantime mentioned there could have been a third event, which would have interrupted the causal chain between the two events. Hence, the first event is not the cause of $\beta\left(t^{2}\right)$ at all, because a causal relation has to be stable (necessary?).

3. If there is a gap between $t^{1}$ and $t^{2}, \alpha\left(t^{1}\right)$ already not exists, before $\beta\left(t^{2}\right)$ starts to exist ("exist", of course, not in the sense of Definition 6 , but rather in the sense of being realized of [11]). Hence $\alpha\left(t^{1}\right)$ cannot be the cause of $\beta\left(t^{2}\right)$, because: how an unexisting event can cause another?

The first objection works on the base of a cause-concept, which deals with full, complete causes. Even if in the meantime another cause exists, according to the suggested approach the causal connection between $\alpha\left(t^{1}\right)$ and $\beta\left(t^{2}\right)$ remains unchanged. Furthermore: just like in case of the third objection the criticism takes for granted, what it argues for - that causal influence cannot pass over gaps in time! Suppose it can, why there should be a third event of the described type? The second objection bases on the idea, that all causal relations are stable (necessary, instances of laws, regularities, ...). This idea is itself objectionable, at least in case of token causation. Furthermore: just like in case of the third objection it ignores the fact, that by assumption $\alpha\left(t^{1}\right)$ and $\beta\left(t^{2}\right)$ occurred. The sentence $\mathcal{U}\left(\alpha\left(t^{1}\right), \beta\left(t^{2}\right)\right)$ can be asserted only post factum, when $\alpha\left(t^{1}\right)$ and $\beta\left(t^{2}\right)$ are known to be existent.

There are no good reasons for forbidding time gaps between causes and effects. But what is about requiring such gaps? At least for more complex events such a requirement is too strong, as it becomes clear after checking some examples.

Keeping apart temporal and causal order can mean several things. It can mean Is it possible to define a causal terminology and a notion of time independent of each other?, and I think it is. It can mean Is it possible, that the ideas of causation and time, given in our experience, are independent of each other?, and here the answer is No.

To give an argument for the first, positive answer, remember the old, well known slot machine: it gives you a ball after you put in a coin. Imagine a slot machine, sometimes giving a ball and sometimes not giving one, as well after you put in a coin as after you did not - this may serve as a model of a possible world without causal relations. The events in that world could be ordered in time, but their coming and going in time is completely independent from other events. Imagine, in contrast, a world without time 
at all, but with a causal structure: hundreds of slot machines, some of them giving a ball, some not, some of them getting a coin, some not; and at a certain place a machine gives a ball just as an effect of putting in a coin in another machine.

Those worlds look very strange, because, I propose, in our world time and causality are related in fact. Hence, my answer to the question about backward causation should be: there are no solid logical arguments against backward causation - so it is logically possible; but there is no case of backward causation, because only relations including a time ordering in the appropriate direction are considered to be causal. Most examples in favor of backward causation are connected with final causes, which are, as far as I know them, easily translated in ordinary forward causal speech. Most arguments against backward causation are connected with loops, or the nonexistence of the cause while the effect already exists, and they, at one hand, ignore the fact that backward causation not automatically means changing the past, and at the other hand they take for granted what should be proved: that there cannot be a backward direction in causation. So I am convinced, that factually there is no backward causation, while logically it is possible.

According to this analysis a causal structure is neither a chain, nor even a tree; it is rather a directed network. It contains nodes with incoming and outgoing edges of different number, but also nodes with only incoming or only outgoing edges and nodes without edges at all (this is, of course, because it is still left open, whether every event has to have causes and/or effects: "every effect has a cause" is a matter of terminology, "every event has a cause" is a matter of philosophy). Direct circles (edges from a node to itself or two edges forward and backward between two nodes) do not exist because of irreflexivity and antisymmetry. Can such a structure induce a time order? It can, under the following conditions:

1. There are no circles in the network.

2. We consider a finite part of the whole network.

The first condition obviously does not exclude backward causation on the network side, it excludes the possibility to change the direction once more: no further forward causation for events, being effects of backward causation. But the intended construction will translate all backward causation on the 
network side into the forward causation equivalent "and then" on the time arrow side. The second condition is not so hard, assumed that even all people together can deal only with a final part of the network. (As becomes clear from the construction, in fact finiteness is required even only on the cause side of the network, so if you believe in the Big Bang being The First Cause, you can take the whole network.) Then, consider the following construction:

\footnotetext{
Interpret a convenient section of the real numbers as time points. Given a finite part of the network described, there are some nodes without incoming edges (they are only causes, or events without causes and effects). Give them any order on an interval of the section and delete them from the network. In the remaining part of the network there are some nodes without incoming edges (they are only causes, or events without causes and effects). Give them any order on another interval of the section, which elements all are greater than any element of the preceding interval, delete the nodes from the network and repeat this step until you come to an end.
}

A natural interpretation consists in: the time points to which the events are assigned are the starting points of the events. For all pairs of events $\alpha$, $\beta$ holds:

If $\mathcal{U}(\alpha, \beta)$, then $\alpha$ is earlier then $\beta$.

The order of causally unconnected events is not totally determined by such a construction. Many different orderings are possible, but the important fact is, that all causal relations point to the same time direction in all of this orderings. So, which of them is the temporal order of events in the empirical world, is a matter of fact.

\section{Necessity and probability}

The following few words now to be told are about a problem, which leads finally to type causation. It is the story of necessity and its weaker variant, probability:

While the notion of causation is often associated with those of necessity and functional dependence, causal expressions often tolerate exceptions, primarily due to missing variables and coarse descriptions. ([9], p. 441)

Suppose a particular causal sentence $\mathcal{U}(\alpha, \beta)$ - what does it mean to say It is necessary, that $\mathcal{U}(\alpha, \beta)$ ? Since we are in the field of token causation, 
$\mathcal{U}(\alpha, \beta)$ is a predicative sentence of form $\mathcal{U} \leftarrow(\alpha, \beta)$ and an usual modal logical necessity ("true in all observable possible worlds") is nonsense. Further, at the end of section 5 was claimed, that a logical connection between the event constituting sentences excludes a causal relation between the events. So the usual interpretation remains: $\mathcal{U}(\alpha, \beta)$ is an instance of a natural law, is of "natural necessity". This idea has been discussed in great detail (cf., for arguments, [2], pp. 93 ff., or [4]), and has been shown false from different points of view. Some objections from the standpoint of this paper are summarized in the following:

1. People also apply causal notions to events, which are unique. In some cases, may be, the causal sentence is the only and single instance in a law, but claiming that for all such sentences exists a law governing it, assumes the required.

2. People apply causal notions in cases, where there is not only no knowledge about a particular law, but where either there is no knowledge about laws at all (in primitive languages, where are not any laws, it is possible to use causality), or where it is doubtful, if there are governing laws (Lady Macbeth's ambitions caused Macbeth's death - which laws are governing that case?).

3. How do we know, that the laws are true? Considering all causal sentences to be instances in laws, and therefore their truth depending on the truth of the laws, that, for its part, should be established independently. I cannot see, how this can avoid a strong realism.

In $[8]$ is an elegant attempt to bypass the usual difficulties:

\footnotetext{
The point is that there is nothing conceptual or logical that would guarantee that the causal connection between $\mathrm{A}$ and $\mathrm{B}$ is at bottom a nomic connection, although in fact it is. That it is in fact a nomic connection is the ontological justification for the experimental method. ([8], p. 69)
}

By repeating the cause event, but with partially other conditions, people try to single out the nomic connection: that property of the event, which (physically) necessitates the effect. This view of an a posteriori connection is less objectionable than that of an a priori identity between token causation and instantiations of laws. Nevertheless, it meets some complaints, too: It requires a very well prepared similarity and identity theory of events, it is submitted to strong realism concerning laws, and, the most important 
point, it rests on the unproved believe in the stability of causal relations: that similar causes really always generate similar effects.

This consideration amounts to doing one of the following: either to acknowledge at least some singular causal sentences expressing contingent facts, or to weaken up the necessity requirements to probability. The former is just the point I want to make, claiming that some singular causal dependencies are instances of causal laws, some are not - that is the topic of the next section. The latter is the way of a still growing community:

In general a probabilistic approach is reasonable only in case of type causation. The main idea consists in claiming, that causes always raise the probability of their effects - which is certainly a sentence about types. A true singular sentence about token causation assumes the existence of both involved events, independent of whether their probability was high or not. Hence, a causal sentence about tokens $\mathcal{U}(\alpha, \beta)$ should be interpreted as follows: " $\alpha$ is of type $\alpha^{*}$, and $\beta$ is of type $\beta^{*}$, and events of type $\alpha^{*}$ always increase the probability of events of type $\beta^{*}$, and $\alpha$ and $\beta$ exist".

The first objection to be made is, that there are cases of increasing the probability which are not causal dependent, and cases of causal dependency which are not increasing the probability (common causes at one hand, and SimPson's paradox at the other). Of course, there are attempts to defend probability analysis against such counterexamples by modifying it, but there is good reason for not to be to sure in obtaining a general solution. The reason is, that causal talk sometimes is used just in order to express a highly improbable, but causal dependency.

An second objection, again, aims at the ontological and epistemological states of facts and laws. Probability approach requires the dependence of statements about token causality on (probabilistic) laws. Hence the observation, that some causal connections are not stable, that sometimes people do not know, whether there is a governing (probabilistic) law ... undermine the whole approach.

\section{Laws and explanations}

The title of that section is misleading insofar as it deals only with causal laws and causal explanations. I cannot propose a general theory of law or explanation, what I am going to do is to show, how it is possible to arrive at laws and explanations based on the ideas above. 
Event types are introduced based on event schemes, general event terms. This provides the opportunity to quantify over general event terms just like over other general terms. The natural idea to express causal laws then is that they claim, that an event of a certain type is always cause of an event of another type, the natural idea to analyze causal explanation is to interpret it as the epistemological counterpart of causation. For laws I will use the formulation mentioned, but nothing seriously changes if is used: An event of a certain type is with a certain probability cause of an event of another type, or another appropriate expression involving probability.

Let $\alpha, \alpha_{1}, \ldots, \beta, \beta_{1}, \ldots$, be event tokens of the common types $\alpha^{*}$ and $\beta^{*}$ respectively, and $O$ be the sentence forming operator "occurs" defined by:

Definition 7. $O(s A) \longleftrightarrow d f A$.

To begin with we discuss strong causal laws:

Definition 8. A strong causal law is a true sentence of form

$$
\Pi \alpha^{*} \Sigma \beta^{*} \mathcal{U}\left(\alpha^{*}, \beta^{*}\right)
$$

There is nothing unusual with such sentences, they are simply generalizations over special cases. Like other generalizations they become false after the occurrence of a counterexample, and usually we get them either by induction, or by deriving them from other general sentences. Especially, there is no logical connection between causes and effects: causal laws may hold in one possible world and do not hold in another. There is no nomic connection between the types independent of the relation between tokens: not because of the law the instances are true, but the other way round.

I propose to call causal dependencies stable, if they are ruled by a strong causal law. The believe, that causal dependencies are stable is expressed by:

For all events $\alpha$ and $\beta$ holds:

$$
\mathcal{U}(\alpha, \beta) \longrightarrow \mathcal{U}\left(\alpha^{*}, \beta^{*}\right)
$$

where $\alpha^{*}$ and $\beta^{*}$ are constructed with the help of appropriate similarity relations (about similarity relations over events cf. [12]). The definitions are chosen in a way, that follows:

For stable causal relations holds: If a relation between event tokens is not governed by a causal law, it is not a causal relation. 
Stability of a causal relation is a property of relations in models, rather than of causal talk in everyday speech. As it was mentioned, causal terminology allows for exceptions, hence we need a weaker notion of a causal law:

Definition 9. A causal law is a true sentence of form

$$
\Pi \alpha^{*} \Sigma \beta^{*}\left(O\left(\alpha^{*}\right) \longrightarrow\left(\sim O\left(\beta^{*}\right) \vee \mathcal{U}\left(\alpha^{*}, \beta^{*}\right)\right)\right)
$$

The basic idea is simple: after an event of type $\alpha^{*}$ the $\beta^{*}$-type event may not occur, but if occurs, it is the effect of $\alpha^{*}$. It is the case of the slot machine; sometimes after putting in a coin you would not get a ball, but if you get it after putting in a coin, it is because of the coin. It seems to me that this is precisely that very way we use general causal sentences in practice. Consider the following example:

\footnotetext{
Reckless driving causes accidents. That means, of course, not that any instance of reckless driving leads to a bad accident - thank fate. It means, that if there is an instance of reckless driving, and an accident too, we are sure about the causal dependence between the latter and the former.
}

The advantages of Definition 9 are bought not for free: there is no conclusion from the causal law and the cause-type event to an effect. It is like in reality - beside the law there may be competing causes, unusual conditions, the whole stock of inconvenient ghosts of causal theory.

Causal explanation exists in two connected steps. It indicates either the search for a cause of an event, or the search for a cause and a causal law. Are there other types of explanation? I think there are, and they differ from causal explanation in explaining other entities: facts, may be sentences, instead of events. It is completely legally to state, that " $3 \times 2 \times 4$ equals 24 " explains, why " $3 \times 8$ equals 24 " - and vice versa. If we want to explain an event, we use causal explanation, and because causal relations are directed, causal explanation is not symmetric:

\footnotetext{
We say that deducing the length of a tower's shadow from the height of the tower, the theory of light propagation, and the elevation of the sun above the horizon explains why the shadow has the length that it has. However, although we could also deduce the height of the tower from the length of the shadow, the theory of light propagation, and the elevation of the sun above the horizon, we would never say that this deduction explains why the tower has the height that it does. ([5], pp. $221 \mathrm{f}$.)
} 
On the level of token causation and causal laws the covering law model works fine. Conversely, explaining event types or requiring stable causal connections (and therefore strong causal laws), the picture no longer remains easy. The explanation of an event henceforth depends not only on the knowledge about a single causal relationship and, may be, a causal law, but also on which part of the data of the world in question is given to the explaining subject.

\section{References}

[1] D. Abusch, Verbs of Change, Causation, and Time, CSLI - Report, 86-50, 1986.

[2] D. Armstrong, What is a Law of Nature, Cambridge University Press, Cambridge, London, New York, New Rochelle, Melbourne, Sydney, 1983.

[3] J. van Benthem, The Logic of Time, Reidel, Dordrecht, Boston, London, 1983.

[4] J. Bigelow and R. Pargetter, Metaphysics of Causation, Erkenntnis 33, 1990, p. 89-119.

[5] V. Hardcastle, Partitions, Probabilistic Causal Laws, and Simpson's Paradox, Synthese 86, 1991, p. 209-228.

[6] H. Hart and A. Honoré, Causation in the Law, Oxford, 1959.

[7] P. Hasle and P. Øhrstrøm, Causal and Temporal Reasoning, Draft, Private Communication, 1991.

[8] A. Heathcote and D. Armstrong, Causes and Laws, Noûs 25, 1991, p. 63-73.

[9] J. Pearl and T. Verma, A Theory of Inferred Causation, Allen, Fikes, Sandevall (Eds.), Principles of Knowledge Representation and Reasoning: Proceedings of the Second International Conference. San Mateo, CA, 1991, p. 441-452.

[10] C. Pizzi, Counterfactuals and the Complexity of Causal Notions, Topoi 9, 1990, p. $147-155$.

[11] U. Scheffler, Events as Shadowy Entities, Konstanzer Berichte Logik und Wissenschaftstheorie 28, 1992.

[12] U. Scheffler, Causal Stability, Svoboda (Ed.) LOGICA '92 - Proceedings, Prague, 1993, p. 132-143.

[13] E. Scheibe, Remarks on the Concept of Cause, Contemporary German Philosophy, Vol. 4, 1984, p. 223-243. 
[14] P. Suppes, A Probabilistic Theory of Causality, Acta Philosophica Fennica XXIV, 1970.

[15] M. Urchs, Kausallogik, Leipzig, 1986.

[16] M. Urchs, On the Logic of Event Causation II (Jaśkowski-systems of Causal Logic), Konstanzer Berichte Logik und Wissenschaftstheorie 26, 1992.

[17] H. Wessel, Logik, Deutscher Verlag der Wissenschaften, Berlin, 1984.

UWE SCHEFFLER

Institute of Philosophy

Humboldt University

Unter den Linden 6

D-10099 Berlin, GERMANY 